\title{
Age-dependent Muscle Adaptation after Chronic Stretch- shortening Contractions in Rats
}

\author{
Erik P. Rader ${ }^{*}$, Kayla N. Layner ${ }^{1}$, Alyssa M. Triscuit ${ }^{1}$, Robert D. Chetlin ${ }^{1,2}$, James Ensey ${ }^{1}$, \\ Brent A. Baker ${ }^{1}$ \\ ${ }^{1}$ Centers for Disease Control and Prevention, National Institute for Occupational Safety and Health, \\ Morgantown, West Virginia 26505, USA \\ ${ }^{2}$ Mercyhurst University, Department of Sports Medicine, Erie, Pennsylvania 16546, USA
}

[Received August 5, 2015; Revised September 18, 2015; Accepted September 20, 2015]

\begin{abstract}
Age-related differences in contraction-induced adaptation have been well characterized especially for young and old rodent models but much less so at intermediate ages. Therefore, additional research is warranted to determine to what extent alterations in adaptation are due to maturation versus aging per se. The purpose of our study was to evaluate muscles of Fisher 344XBrown Norway rats of various ages following one month of exposure to stretch-shortening contractions (SSCs). With exposure, muscles mass increased by $\sim 10 \%$ for 27 and 30 month old rats vs. $\sim 20 \%$ for 3 and 6 month old rats $(P<0.05)$. For 3 month old rats, maximum isometric force and dynamic peak force increased by $22 \pm 8 \%$ and $27 \pm 10 \%$, respectively $(P<0.05)$. For 6 month old rats, these forces were unaltered by exposure and positive work capacity diminished by $27 \pm 2 \%(P=0.006)$. By 30 months of age, age-related deficits in maximum isometric force, peak force, negative work, and positive work were apparent and SSC exposure was ineffective at counteracting such deficits. Recovery from fatigue was also tested and exposure-induced improvements in fatigue recovery were indicated for 6 month old rats and to a lesser extent for 3 month old rats whereas no such effect was observed for older rats. Alterations in fatigue recovery were accompanied by evidence of substantial type IIb to IIx fiber type shifting. These results highlight the exceptional adaptive capacity for strength at a young age, the inclination for adaptation in fatigue recovery at early adulthood, and diminished adaptation for muscle performance in general beginning at late adulthood. Such findings motivate careful investigation to determine appropriate SSC exposures at all stages of life.
\end{abstract}

Key words: dorsiflexor muscles, dynamometer, Fisher 344XBrown Norway rats, repetitive exposure

With aging, muscle mass and strength decreases of $~ 30 \%$ are sustained by 80 years of age [1,2]. Exercise is recommended to offset this decline but research regarding specific details concerning training design and parameters remain ongoing [2,3]. Furthermore, the adaptive capacity to training appears to be blunted for the elderly thereby making training design more difficult $[4,5]$. Stretchshortening contractions (SSCs), contractions in which muscle is activated for both the initial stretch and subsequent shortening, are common in daily activities and exercise consisting of SSCs has been proposed as an effective training modality at advanced age $[6,7]$. To investigate the effects of chronic exposure to SSCs at a fundamental level, we developed an in vivo experimental rat model in which the dorsiflexor muscles of the hind limb were exposed to SSCs three times per week for one month [8]. For young rats, such exposure induces a 20\% gain in muscle mass and muscle performance [8-10]. For old rats, the same exposure induces muscle mass gains of less than $10 \%$ accompanied by unaltered or even decreased performance measures [8-10].

Recent reports regarding aging have demonstrated that the aging process is apparent earlier than previously proposed [11, 12]. The results of these studies 
demonstrate that indicators of the rate of aging can be detected long before the onset of chronic disease [11]. Physical capacity including balance and strength appears to be sensitive to aging rate throughout adulthood $[11,12]$. These findings provide the motivation to investigate intermediate ages between youth and old age, especially in regards to muscle performance. Since such an age range has not been investigated regarding SSC-induced adaptation, questions remain as to how early in life adaptive capacity begins to change and which performance outcomes are most age-sensitive.

To address the gap in our understanding of adaptive capacity to SSCs at various ages, the purpose of the present study was to characterize performance alterations to chronic SSC exposure for several age groups. Fischer 344 Brown Norway hybrid rats aged 3, 6, 27, and 30 months of age were subjected to one month of SSC exposure and isometric force, dynamic peak force, negative work, and positive work were evaluated. To determine underlying SSC-induced modifications, fiber type analysis was performed. The findings are valuable for establishing how early in adulthood adaptive responses are altered and the nature of that alteration important information when considering the appropriate exposure to contractions for distinct age groups.

\section{METHODS AND MATERIALS}

\section{Animals}

Male Fischer Brown Norway hybrid rats (F344 X BN F1) were obtained from the National Institutes of Aging colony. Rats 3, 6, 27, and 30 months old were housed in an Association for Assessment and Accreditation of Laboratory Animal Care (AAALAC)-accredited animal quarters. All animal procedures were approved by the Animal Care and Use Committee at the National Institute for Occupational Safety and Health in Morgantown, WV.

\section{SSC chronic exposure}

The SSC exposure was based on a previously described procedure in which rats were exposed to 80 SSCs 3 times per week for 4.5 weeks [8]. For each exposure, rats were anesthetized with isoflurane gas and placed supine on a heated $x-y$ positioning table. The left knee was secured in flexion at $90^{\circ}$ with a knee holder and the left foot secured to a fixture containing a load cell. For activation of the dorsiflexor muscles, platinum electrodes were placed subcutaneously in the region of the common peroneal nerve. All stimulation was set at optimal parameters for maximal contraction; 4-V magnitude, $0.2-\mathrm{ms}$ pulse duration, and 120-Hz frequency [13].
To assess static performance, dorsiflexor muscles were exposed to a maximal isometric tetanic contraction for $300 \mathrm{~ms}$ with the ankle at $90^{\circ}$. After a 2-minute interval, dynamic performance was assessed by exposure to a single SSC test consisting of maximally activating the dorsiflexor muscles for $300 \mathrm{~ms}$ at ankle angle of $70^{\circ}$, then rotating the ankle from $70^{\circ}$ to $140^{\circ}$ at $500^{\circ}$ per second, and then returning to $70^{\circ}$ at the same velocity. Activation continued for an additional $300 \mathrm{~ms}$ after cessation of ankle rotation. The maximum force during the stretch phase was referred to as peak force. Negative work was the work required to stretch the muscle while positive work was the work done by the muscle during the shortening phase. Two minutes following the single SSC test, the dorsiflexor muscles were then exposed to the $80 \mathrm{SSC}$ protocol. The protocol consisted of 8 sets of SSCs with 2-minute intervals between sets and 10 SSCs per set with 2 -second intervals between SSCs. For each SSC, while the muscles were maximally activated, the ankle was set to $90^{\circ}$ for $100 \mathrm{~ms}$, then rotated to $140^{\circ}$ at $60^{\circ} \%$, returned to $90^{\circ}$ at the same velocity, and lastly, the muscles were deactivated $300 \mathrm{~ms}$ later. The velocity of the SSCs of $60^{\circ}$ per second was chosen to promote performance adaptation and hypertrophy rather than injury as was found at a higher velocity $\left(500^{\circ}\right.$ per second) in other studies [8]. Indeed, only minor levels of muscle inflammation and degeneration are evident by histological analysis for young and old rats in the days to weeks following the SSC exposure utilized in the present study $[8,9,14]$. Two minutes after the $80 \mathrm{SSCs}$, isometric force was measured at the same settings as those for the maximum isometric tetanic contraction and four minutes after the 80 SSCs, dynamic performance was measured using the same single SSC test as that used prior to the 80 SSCs. These post-protocol isometric and dynamic measures were used to assess recovery from fatigue. To assess the effect of chronic SSC exposure, the force and work outputs were averaged for the sessions during the first and last week of exposure to determine initial and final values.

At the end of the 4.5 weeks of SSC exposures, TA muscles were surgically removed, weighed, and the tibia length recorded. The mid-belly of the TA muscle was covered with tissue freezing media and immersed in isopentane at $-80{ }^{\circ} \mathrm{C}$ for histology. Normalized muscle mass was determined by dividing the muscle mass by tibia length. Final muscle quality was quantified as maximum isometric tetanic force for the final week of exposure divided by normalized muscle mass. In a previous study regarding body masses and non-exposed muscles of 3 month old F344 X BN F1 rats $(N=30)$, body masses, muscle masses, tibia lengths, and normalized muscle masses were $326 \pm 5 \mathrm{~g}, 605 \pm 10 \mathrm{mg}, 40.3 \pm 0.2 \mathrm{~mm}$, and $15.0 \pm 0.2 \mathrm{mg} / \mathrm{mm}$, respectively [14]. These values were 
not significantly different from those at 4 months of age (i.e. one month after exposure began at 3 months of age) in the present study (Table 1 and Figure 1A). This confirmed that the chronic SSC exposure duration of 4.5 weeks was short enough so that growth due to normal maturation was not a confounding factor during that time period.

Table 1. Body masses, muscle masses, and tibia lengths for rats of various ages for control and SSC-exposed conditions.

\begin{tabular}{|c|c|c|c|c|c|}
\hline & & \multicolumn{4}{|c|}{ Age (months) } \\
\hline & & 3 & 6 & 27 & 30 \\
\hline \multirow[t]{2}{*}{ Body mass (g) } & Initial & $305 \pm 23$ & $402 \pm 6^{+}$ & $554 \pm 13 \dagger \neq$ & $529 \pm 20 \nmid \neq$ \\
\hline & Final & $321 \pm 22$ & $373 \pm 6^{*+}$ & $508 \pm 10^{*}+\neq$ & $462 \pm 15^{*} \dagger \neq$ \\
\hline \multirow[t]{2}{*}{ Muscle mass (mg) } & Contralateral & $622 \pm 38$ & $666 \pm 18$ & $712 \pm 11$ & $578 \pm 27$ \\
\hline & Exposed & $751 \pm 57^{*}$ & $772 \pm 27 *$ & $770 \pm 14^{*}$ & $645 \pm 38 * \neq \neq \S$ \\
\hline \multirow[t]{2}{*}{ Tibia length (mm) } & Contralateral & $40.6 \pm 0.6$ & $42.7 \pm 0.2 \dagger$ & $44.7 \pm 0.2 \dagger \neq$ & $44.6 \pm 0.7 \dagger \ddagger$ \\
\hline & Exposed & $40.7 \pm 0.6$ & $42.7 \pm 0.3 \dagger$ & $44.6 \pm 0.2 \dagger^{\prime}$ & $44.7 \pm 0.7 \dagger \ddagger$ \\
\hline
\end{tabular}

Body masses were measured the initial and final day of the 1 month of SSC exposure. Muscle masses and tibia lengths were measured for contralateral control and exposed muscles. Values are means \pm S.E.M. *Different from initial or contralateral value; $\uparrow$ Different from value for 3 month old rats; ¥Different from value for 6 month old rats; §Different from value for 27 month old rats, $P<0.05$.

\section{Fiber type analysis}

The mid-belly of each TA muscle was transversely cryosectioned at $10 \mu \mathrm{m}$ thickness followed by immunofluorescence MHC staining. The staining protocol was based on a previously described multicolor immunofluorescence method [15]. Sections were allowed to air dry for 15 minutes, rehydrated in PBS for 5 minutes, and then blocked with $10 \%$ goat serum in PBS for one hour at room temperature. The sections were then incubated in the following primary antibody cocktail overnight at $4^{\circ} \mathrm{C}-$ antibodies against MHCI (BA-F8; 1:10), MHCIIa (SC-71; 1:200), MHCIIb (BF-F3;1:200), and laminin (L9393;1:400). The antibodies against the MHC isoforms were purchased from Developmental Studies Hybridoma Bank (University of Iowa) and the antibody against laminin was purchased from Sigma. After washes in PBS, the sections were incubated in a cocktail of secondary Alexa Fluor antibodies (Life Technologies) for two hours at room temperature -350 IgG2b goat anti mouse (A21140; 1:250), 594 IgG1 goat anti-mouse (A21125; 1:100), 488 IgM goat anti-mouse (A21042; 1:500), and 488 IgG goat anti-rabbit (A11008; $1: 500)$.

Fiber type analysis was performed by a standardized stereological method [8]. The midpoint of the muscle section was identified, and stereological analysis was systematically repeated at 5 equally spaced sites across the section on each side (by $1 \mathrm{~mm}$ ) of the midpoint. At each site, points of a 121-point 11-line overlay graticule $\left(0.04 \mathrm{~mm}^{2}\right.$ square with 100 divisions $)$ were evaluated at $20 \mathrm{X}$ magnification. Since 121 points were evaluated in 10 fields, a total of 1210 points were analyzed per muscle section. Each point was identified as overlaying a MHCI (blue), MHCIIa (red), MHCIIb (green), MHCIIx (lacking staining), or interstitium. Percent of muscle tissue comprised of each fiber type and interstium was computed as the percentage of points which overlaid each component relative to the total number of points. In addition to evaluating points of the overlay graticule, the number of muscle fibers corresponding to each fiber type were counted within the 
boundary of the graticule. Fibers were counted when the topmost point of the fiber perimeter was within the graticule boundary. The number of fibers per unit crosssectional area (number of fibers per $\mathrm{mm}^{2}$ ) was calculated as the total number of fibers counted divided by the total area sampled over the 10 regions (i.e. $0.4 \mathrm{~mm}^{2}$ ). The percentage of each fiber type was determined by dividing
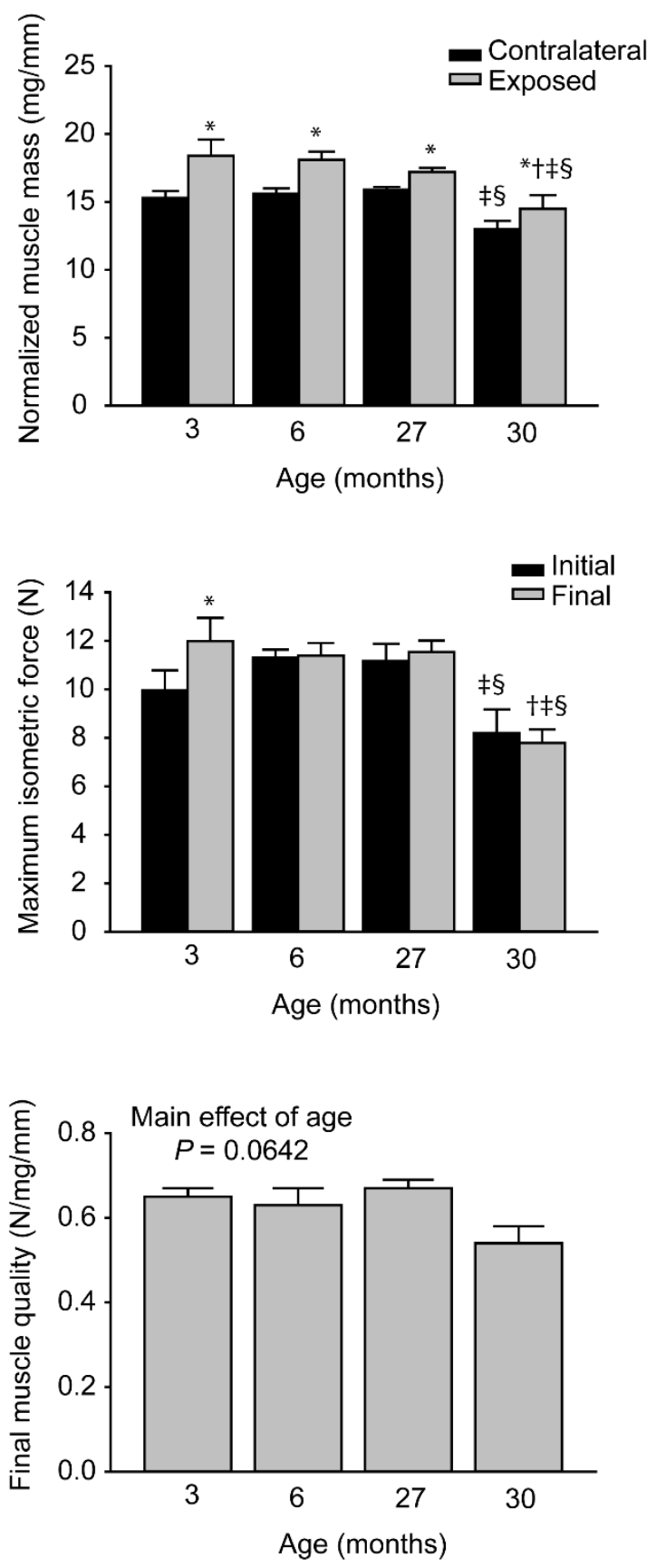

the number of fibers corresponding with each fiber type by the total number of fibers counted. Mean muscle fiber area $\left(\mu \mathrm{m}^{2}\right)$ for a particular fiber type was determined by dividing the percent of tissue comprised of that fiber type by the appropriate fiber number per unit area.

Figure 1. Muscle mass and isometric performance measures following chronic SSC exposure were age-dependent. For both the exposed and contralateral control TA muscles, muscle mass was normalized by tibia length. Maximum isometric forces were assessed for the initial and final weeks of SSC exposure. Final muscle quality was calculated for exposed muscles by dividing the final maximum isometric force value by normalized muscle mass. Sample sizes were $N=5$ per group. Values are means \pm S.E.M. *Different from non-exposed value (i.e. contralateral muscle in context of muscle mass or initial data in context of maximum isometric force and muscle quality); $\dagger$ Different from value for 3 month old rats; $\$$ Different from value for 6 month old rats; §Different from value for 27 month old rats, $P<0.05$. 


\section{Statistical Analysis}

Data were analyzed using ANOVA with the variable of animal identification as a random factor to account for repeated measures within animal when appropriate. Post hoc comparisons were performed using Fisher's least
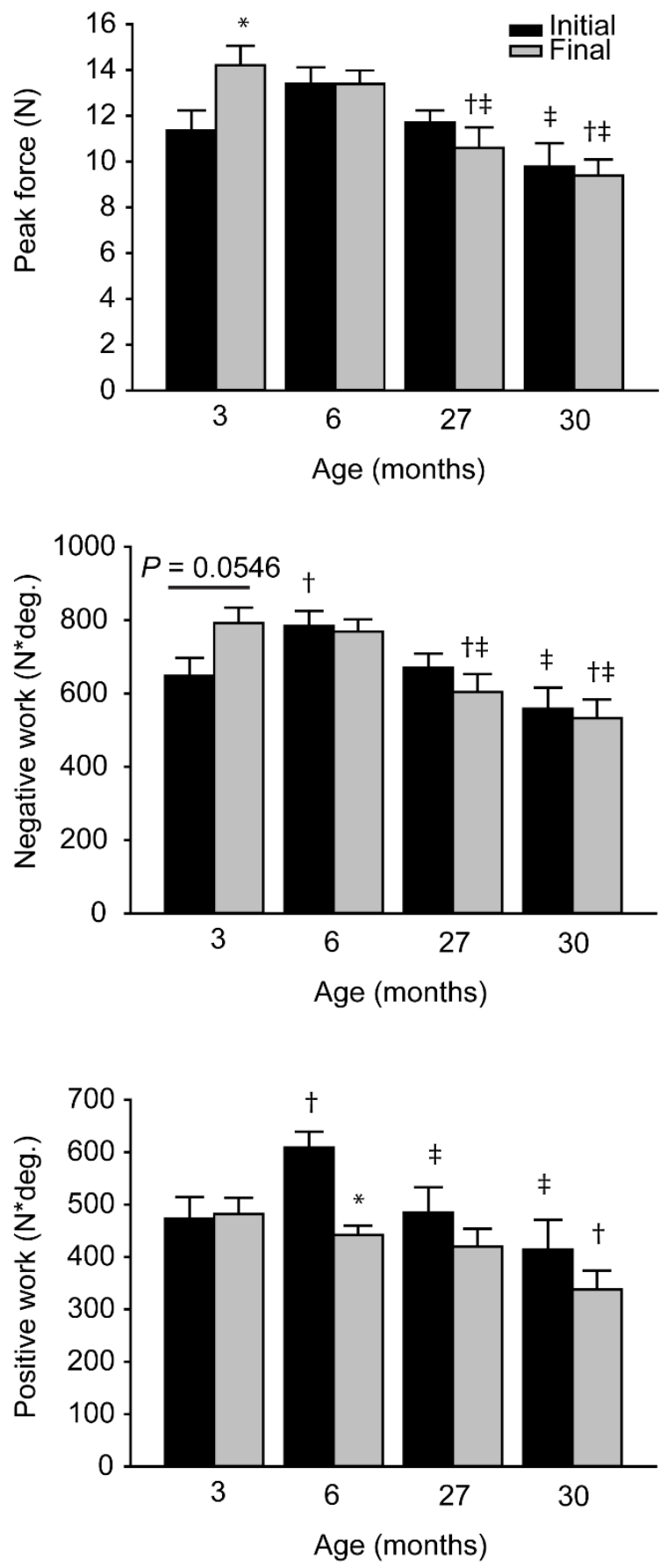

significant difference method. All data are shown as means \pm S.E.M. $P<0.05$ was considered statistically significant.
Fig. 2. Chronic SSC-induced alterations in dynamic muscle performance were sensitive to aging. Measures of peak force, negative work, and positive work were evaluated for the single SSC test prior to the 80 contraction protocol in the initial and final weeks of exposure. For 3 month old rats, chronic SSC exposure increased peak force and a trend for an increase in the magnitude of work required to stretch muscle (i.e. negative work) was observed. For 6 month old rats, SSC exposure decreased the capacity for work done by the muscle during shortening (i.e. positive work). Sample sizes were $N=5$ per group. Values are means \pm S.E.M. *Different from initial value; $\dagger$ Different from value for 3 month old rats; $\downarrow$ Different from value for 6 month old rats; $\S$ Different from value for 27 month old rats, $P<0.05$. 


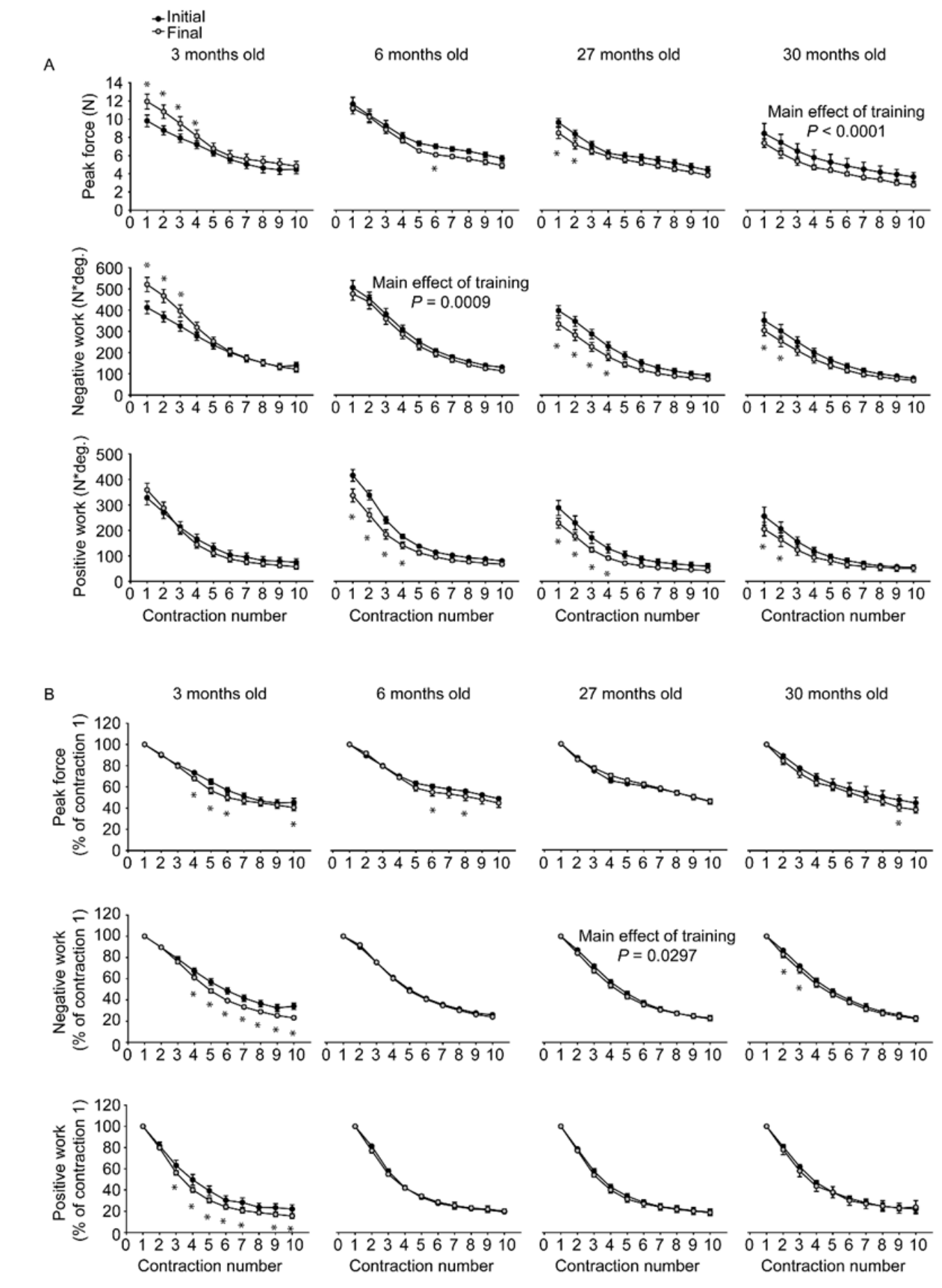

Figure 3. Age differential effects of chronic SSC exposure on SSC-induced fatigue were present. Values for peak force, negative work, and positive work were evaluated for the first set of 10 contractions for the SSC sessions of the initial and final weeks. (A) Analysis of the absolute values demonstrated that muscles of 3 month old rats either improved or maintained absolute performance while muscles of the older groups generated reduced values. (B) To evaluate fatigue in isolation, peak force, negative work, and positive work values were normalized to the value of the first contraction. Chronic SSC exposure decreased fatigue resistance for all of the outcomes for 3 month old rats and depending on the performance outcome, had either no effect or diminished fatigue resistance for the older groups. Sample sizes were $N=5$ per group. Values are means \pm S.E.M. *Different from initial value, $P<0.05$. 


\section{RESULTS}

The 3 month old (and to a lesser extent, the 6 month old rats) were in the process of maturation as evident by smaller tibia lengths and body weights relative to 27 and 30 month old rats (Table 1). In addition, contralateral muscle masses of 3 month old rats exhibited a trend $(P=$ 0.0552 ) for smaller values relative to those for 27 month old rats (Table 1). Sarcopenia was evident for 30 month old rats and SSC-induced muscle mass gains of diminished magnitude with aging (Table 1, Figure 1). Normalized muscle masses for contralateral muscles of 30 month old rats were $10 \%$ less than those for 6 and 27 month old rats $(P<0.05)$. When considering exposed muscles relative to contralateral muscles, SSC-induced normalized muscle mass increases of $20 \pm 3 \%$ and $16 \pm$ $2 \%$ for 3 month old and 6 month old rats, respectively. Muscles for the older age groups increased muscle mass by a smaller extent, $8 \pm 1 \%$ for 27 month old rats (different from 3 and 6 month old values, $P<0.05$ ) and $11 \pm 2 \%$ for
30 month old rats (different from 3 month old values, $P=$ 0.0251).

Chronic SSC exposure improved static performance exclusively for 3 month old rats. Static performance was assessed by maximum isometric tetanic force in the initial and final phases of exposure. Initially, muscles of 30 month old rats generated forces that were $30 \%$ less $(P<$ $0.05)$ than those for 6 and 27 month old rats (Figure 1). With SSC exposure, isometric force increased by $22 \pm 8 \%$ for 3 month old rats $(P=0.0327)$. No such adaptation was observed at 6,27, or 30 months of age. At the end of chronic SSC exposure, the values of isometric force were divided by normalized muscle mass to determine muscle quality. Analysis of the muscle quality data indicated that the muscle quality for 30 month old rats was $\sim 15 \%$ less than those of other age groups, $P=0.0642$ (Figure 1). Since, at 30 months of age, SSCs induced a muscle mass gain without a rise in isometric force, the implication is that exposure exacerbated the deficiency in muscle quality observed at this age.
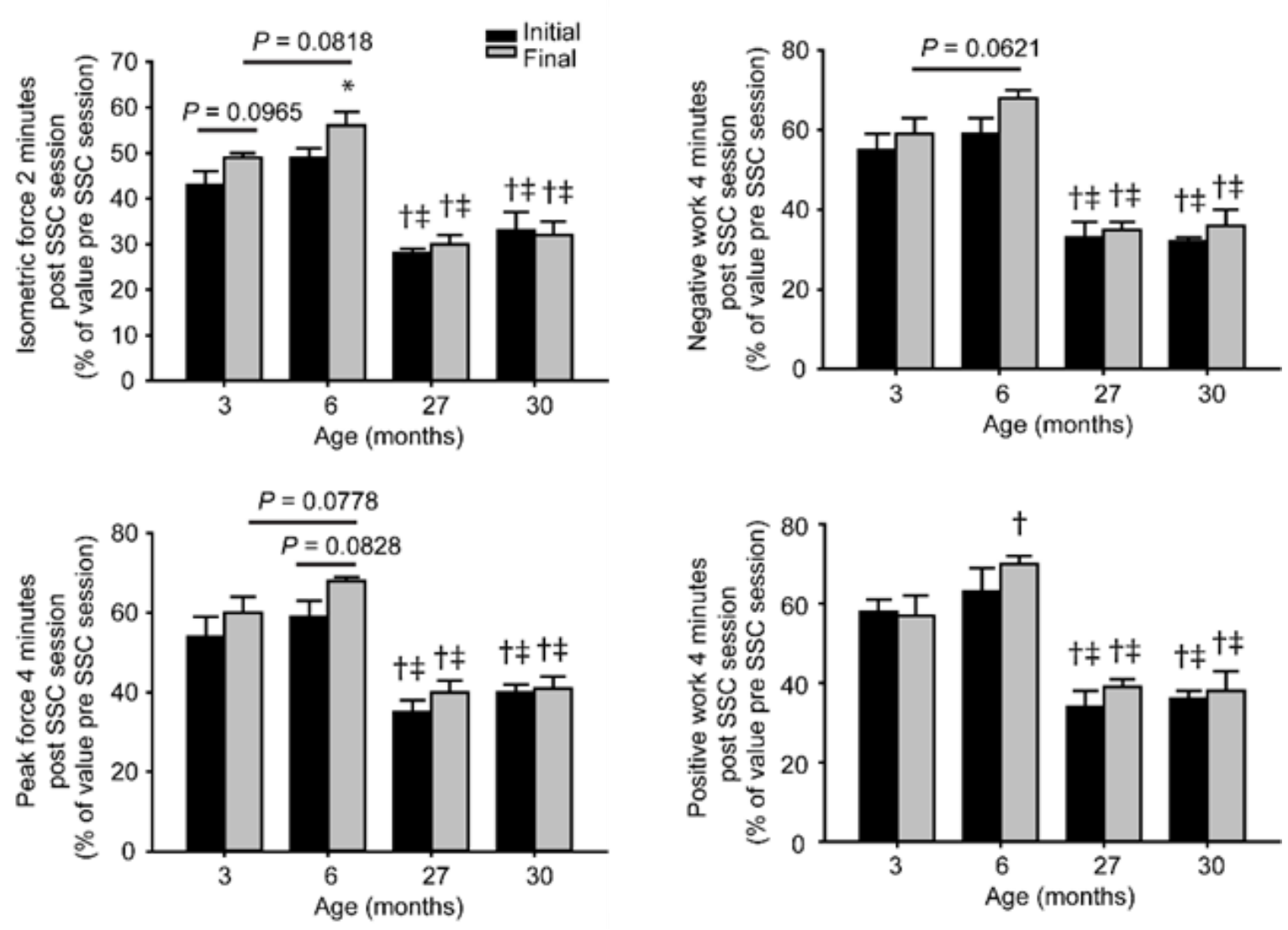

Figure 4. Indication of SSC-induced improvements in recovery from fatigue for the younger age groups. For the initial and final weeks of SSC exposure, isometric forces were evaluated 2 minutes after each 80 contraction session and expressed relative to the pre-session maximum isometric force. A single SSC test was also performed 4 minutes after each 80 contraction session to assess peak force, negative work, and positive work and expressed relative to these values prior to each session. Sample sizes were $N=5$ per group. Values are means \pm S.E.M. *Different from initial value; $\uparrow$ Different from value for 3 month old rats; $\$$ Different from value for 6 month old rats, $P<0.05$. 


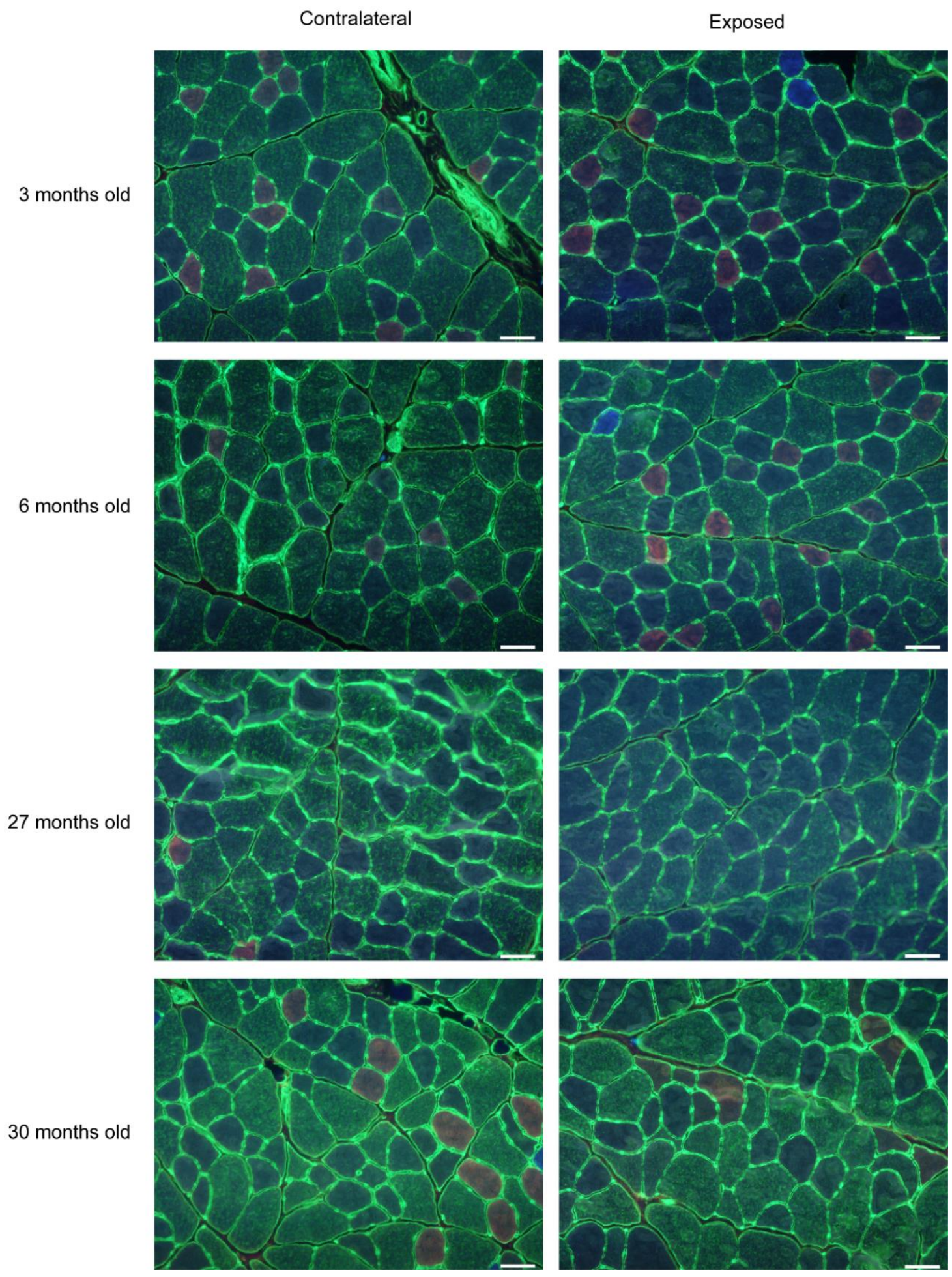

Figure 5. MHC immunofluorescence labeling was apparent in transverse sections of contralateral and exposed TA muscles following chronic SSC exposure. MHC immunofluorescence labeling was apparent for laminin (green) and multiple MHC isoforms - I (blue), IIa (red), IIb (green), and IIx (negative for staining) - in transverse sections of contralateral and exposed TA muscles following chronic SSC exposure. Scale bar $=50 \mu \mathrm{m}$. 
Dynamic performance of 3 month old rats improved with chronic SSC exposure whereas SSC-induced deficits were apparent at 6 months of age and older. Age-related deficiencies in several measures of the single SSC test were observed prior to exposure to SSCs (Figure 2). At 30 months of age, peak force, negative work, and positive work were $30 \%$ less than those for 6 month old rats $(P<$ $0.05)$. At 27 months of age, positive work was $20 \%$ less than values for 6 month old rats $(P=0.0289)$. Initial positive and negative work values at 3 months of age also were not at 6 month levels indicating that these outcomes become optimal only upon reaching adulthood. Chronic SSC exposure induced an increase in peak force by $27 \pm$ $10 \%(P=0.0255)$ and a trend for greater negative work capacity $(P=0.0546)$ in muscles of 3 month old rats (Figure 2). No such exposure effect was observed at older ages. Rather, evidence for SSC-induced deficits were present for 6, 27, and 30 month old rats. Positive work was the most age-sensitive performance measure following SSC exposure with a $27 \pm 2 \%$ decrease apparent by 6 months of age $(P=0.0057)$. SSC-induced decrements in peak force and negative work occurred later by 27 months. This was supported by the observation that age-related differences with 6 month old values became apparent only after SSC exposure (Figure 2).

To determine the effect of SSCs on fatigue resistance, peak forces and work values during the first set of SSC contractions in the initial and final weeks of exposure were analyzed. For 3 month old rats, chronic SSC exposure increased the peak forces and negative work values of the first 3 to 4 contractions and then values returned to initial levels, $P<0.05$ (Figure 3A). This increased performance in absolute terms was accompanied by decreased fatigue resistance when considering performance relative to that for the first contraction (Figure 3B). This finding was not surprising given that the SSC protocol was designed to induce strength and muscle mass gains rather than improve fatigue resistance. The chronic SSC exposure-induced effect at 6,27, and 30 months of age was more severe in that all of the absolute performance measures decreased and in several cases fatigue-resistance declined as well (Figure 3A-B).

Performance measures shortly after each 80 SSC protocol indicated that recovery from fatigue was improved after chronic SSC exposure for 6 month old rats and to a lesser extent for 3 month old rats. In the initial and final weeks of exposure, static performance was assessed by isometric force measurement 2 minutes after each SSC session and dynamic performance was assessed by the single SSC test 4 minutes after each SSC session. These measures were then expressed as a percentage of pre-SSC session values as an indicator of fatigue recovery (Figure 4). Even in the absence of chronic SSC exposure (i.e. as evaluated in the initial week), aging had a profound effect on fatigue recovery. Muscles of 27 and 30 months of age recovered to $\sim 50 \%$ of the extent of that of younger rats for all of the performance measures. With chronic SSC exposure, isometric force recovery improved by $14 \%$ for both 3 month old rats (trend, $P=0.0965$ ) and 6 month old rats $(P=0.0461)$. Fatigue recovery for peak force, negative work, and positive work improved by $11 \%$ to $15 \%(P<0.05)$ for 6 month old rats while these dynamic measures were unaltered for 3 month old rats. No exposure effect was observed for any of the static and dynamic performance outcomes evaluated for the 27 and 30 month old rats.

To investigate a potential mechanism for enhanced fatigue recovery after chronic SSC exposure, fiber type analysis was performed (Figure 5). For contralateral control muscles, type IIa fibers of 30 month old rats were $\sim 45 \%$ greater in cross-sectional area than values at 3 and 6 months of age, $P<0.05$ (Figure 6A). The percentage of type IIa fibers decreased by $30 \%$ to $50 \%$ at older ages ( 27 and 30 months of age) relative to that at young age ( 3 and 6 months of age), $P<0.05$ (Figure 6B). Also for contralateral control muscles, an age-related $60 \%$ to $70 \%$ increase in type IIx fiber percentage was observed at 27 and 30 months age relative to the younger age groups. The percent of muscle tissue comprised of interstitium and each fiber type was also analyzed. The interstitium expressed as percent of tissue for contralateral control muscles vs. exposed muscles was $6.0 \pm 0.8 \%$ vs. $7.8 \pm$ $1.1 \%$ for the 3 month old group, $7.4 \pm 1.0 \%$ vs. $8.0 \pm 0.9 \%$ for the 6 month old group, $7.2 \pm 1.3 \%$ vs. $7.8 \pm 1.0 \%$ for the 27 month old group, and $10.3 \pm 0.9 \%$ vs. $8.3 \pm 0.8 \%$ for the 30 month old group (ANOVA was not significant - smallest $P$ value was for the age x exposure interaction; $P=0.1238$ ). When the Type IIb fibers were expressed as a percentage of tissue, the 30 month old group had lower values than those for 3 and 6 month old rats $(P<0.05)$. With exposure, type IIa and IIx fibers were the most responsive to increases in fiber cross-sectional area for all the age groups with the exception of the 30 month old rats (Figure 5B). Chronic SSC exposure also induced a decrease in the type IIb fiber percentage (relative to total number of fibers) for all the age groups with 6 month old rats exhibiting the most extreme reduction of $70 \%$ (Figure 6B). Decrease in type IIb percentage was accompanied by an increase in percentage of type IIx fibers of $60 \%$ for 3 month old rats and $86 \%$ for 6 month old rats. No significant SSC-induced increases in type IIx fiber percentage were observed for the older groups. When fiber type was expressed as percentage of tissue, 6 month old rats exhibited the most robust alterations and were the only group to exhibit SSC-induced changes in three fiber types - type IIa, IIx, and IIb fibers (Figure 6C). Overall, the fiber type analysis revealed that alterations in fiber 
type distribution attributable to chronic exposure to SSCs accompanied the improved recovery from fatigue observed at younger ages, especially at 6 months of age.
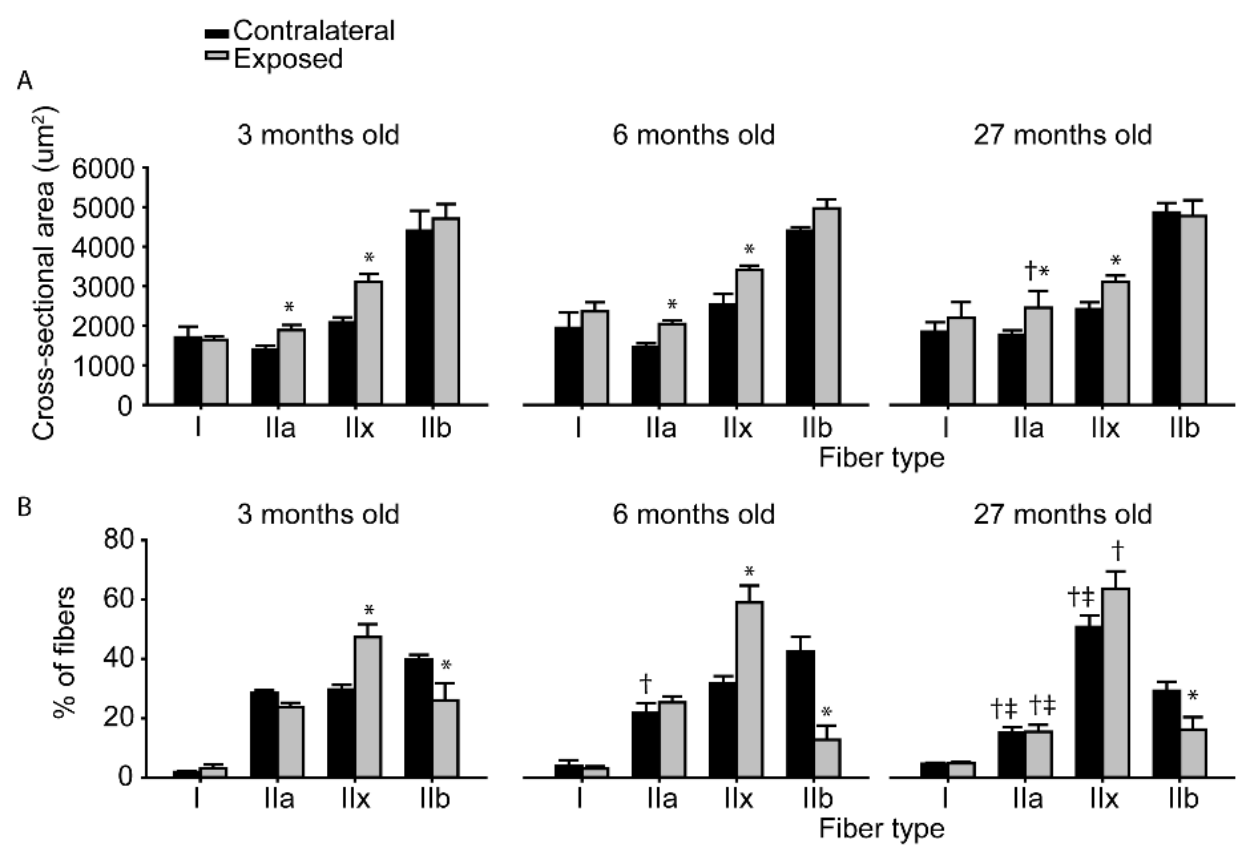

27 months old

30 months old

C
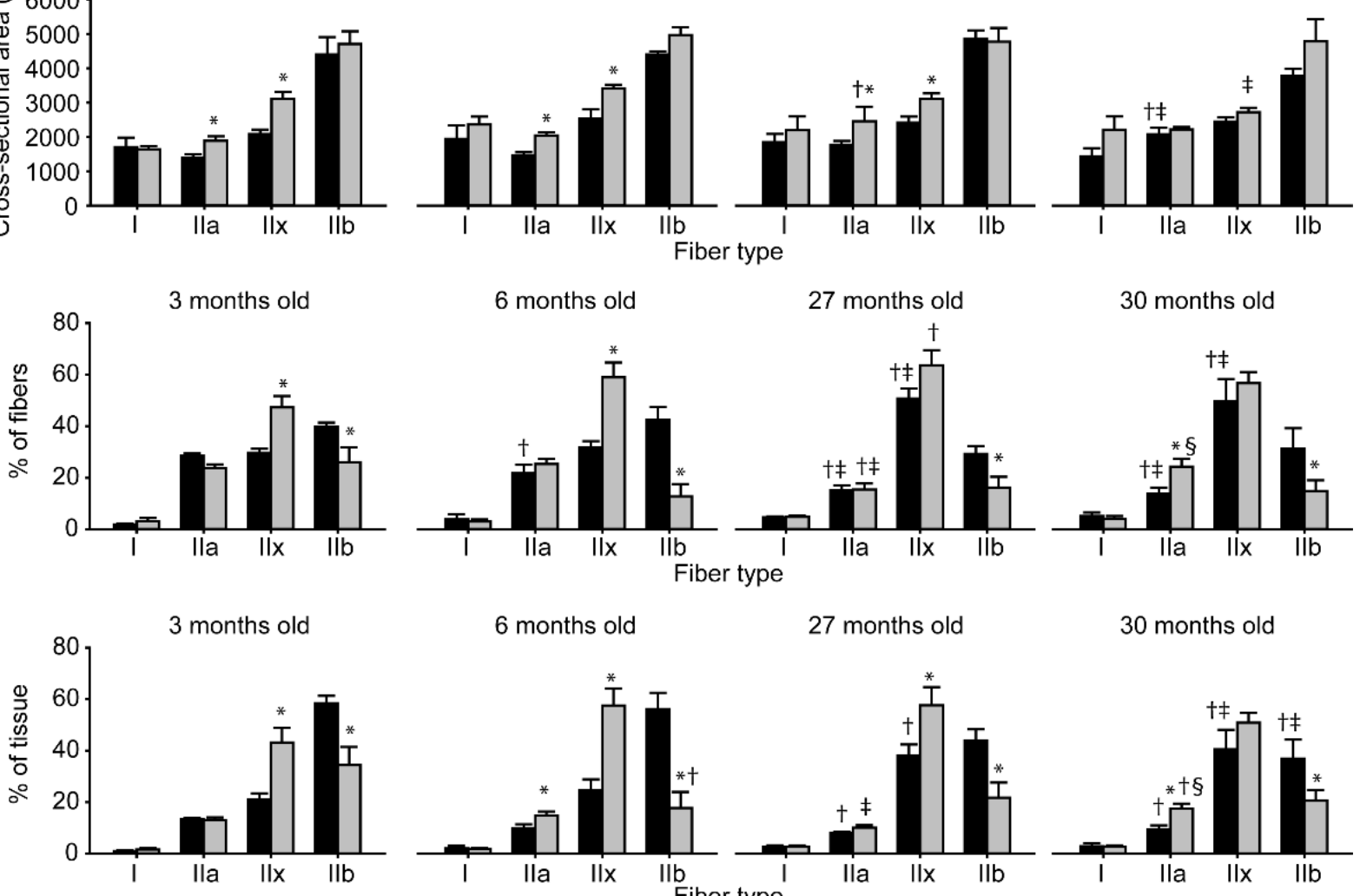

6 months old

27 months old

30 months old
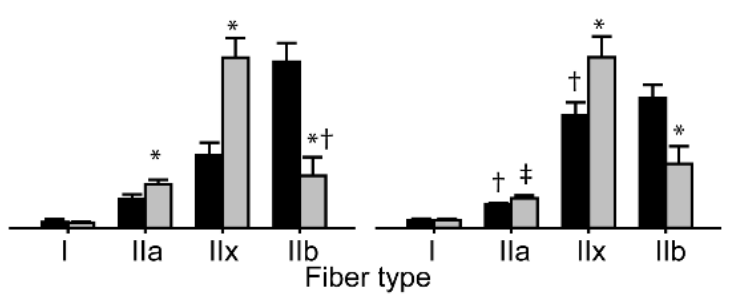

30 months old

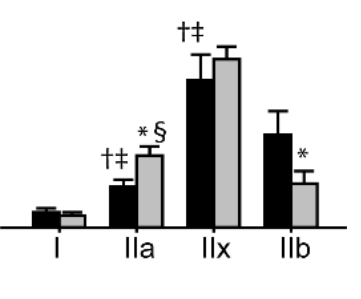

Figure 6. Pronounced alterations in type IIb and IIx fiber type distribution were apparent for 3 and 6 month old rats but not at older ages after chronic SSC exposure. (A) Analysis of muscle fiber cross-sectional area for exposed and contralateral non-exposed muscles indicated that increased area occurred predominantly for type IIa and IIx fibers for all the age groups with the exception of 30 month old rats. (B) With exposure, the coupling of a decreased percentage of type IIb fibers with increased percentage of type IIx fibers occurred exclusively for 3 and 6 month old rats. (C) When fiber type percentage is expressed relative to the tissue, 6 month old rats were the only age group to exhibit SSC-induced changes in all type II fibers - IIa, IIx, and IIb fibers. Sample sizes were $N=5$ per group. Values are means \pm S.E.M. *Different from initial value; $\uparrow$ Different from value for 3 month old rats; $\$$ Different from value for 6 month old rats, $P<0.05$.

\section{DISCUSSION}

Age-dependent alterations in response to chronic exposure to contractions have been observed for human subjects and animal models previously [4, 5, 8, 10]. However, these studies were limited in the number of age groups studied. In the present study utilizing an experimental rat model, we demonstrate that age-related differences in muscle adaptation begin early in adulthood.
While isometric and peak force increased by $20 \%$ to $30 \%$ for young rats ( 3 months old), these forces for young adult rats (6 months old) were unaltered. Furthermore, positive work capacity decreased with chronic SSC exposure for 6 month old rats. Rather than accomplishing the main goal of the SSC exposure (i.e. increased strength), muscles of 6 month old rats improved in the ability to recover from fatigue. This fatigue recovery was accompanied by a decrease in tissue composition for type IIb fibers and 
increases for type IIx and IIa fibers. At late adulthood (27 months of age) and old age (30 months of age), all of the SSC-induced performance and fiber type alterations were muted relative to those for younger rats. These findings demonstrate a high capacity for strength adaptation at a young age, the propensity for SSC-induced improvements in fatigue recovery at early adulthood, and the onset of greatly diminished adaptation beginning at late adulthood.

Age-dependent responsiveness to resistance-type training is well documented for extreme age groups. Elderly are capable of responding to training with gains in muscle mass and performance but to a lesser extent compared with that of young adults $[4,5,16,17]$. For muscles of old rats exposed to the SSC protocol utilized in the present and previous studies, SSC-induced increases in muscle mass were blunted and performance gains were not observed $[8,18,19]$. The compromised response at advanced age is accompanied by heightened oxidative stress and deficiencies in the heat shock protein response - proteins essential for adaptation to stress and protein synthesis [19]. Following SSC exposure, muscles of old rats exhibit diminished oxidant buffering capacity, high levels of hydrogen peroxide, and reduced levels of heat shock proteins $[10,18,19]$. The role of these factors in the age-dependent response is confirmed by the finding that Vitamin E and Vitamin C supplementation increases the oxidant buffering capacity, decreases hydrogen peroxide levels, and improves adaptation in performance for old rats [18].

The present study furthers our previous work by demonstrating that chronic contraction-induced performance gains are attenuated not only at advanced age but also at early adulthood. While muscles of 3 month old rats gained by $22 \%$ in maximum isometric force and by $27 \%$ in peak force with SSC exposure, muscles of 6 month old rats did not exhibit any performance gains and positive work capacity even decreased. The capacity to perform positive work appears to be especially sensitive to SSCinduced maladaptation even at old age [8]. This finding is consistent with studies which report that power output is affected with age earlier than other performance outcomes such as static performance (i.e. isometric force) [20-22]. Rats at 3 months of age were in a late adolescent state of maturation as confirmed by $5 \%$ shorter tibia lengths and 24\% lower body weights relative to those of 6 month old rats. Several studies have reported increases in strength and muscle size after chronic exposure to contractions are possible at adolescence [8, 23-25]. Experiments requiring 3 week old rats to climb with tail weights demonstrated that 2 months of such sessions elicited performance gains and increases of $\sim 15 \%$ in mean muscle fiber cross-sectional area relative to values for sedentary rats $[23,24]$. In human studies, studies report that responsiveness to exercise may even be optimal during adolescence and the transition from adolescence to adulthood [26, 27]. For old rats, the mechanism for the diminished adaptive capacity in performance despite modest gains in muscle mass may relate to oxidative stress and a compromised heat shock protein response [10, 18, 19]. With aging, antioxidant supplementation with Vitamin E and C enables SSC-induced performance gains especially for positive work implying an appropriate redox environment as a key factor in maintaining force during individual contractions [18]. Whether oxidative stress underlies the distinction between the 3 month old rats and the older age groups (beginning at 6 months of age) in the present study is not known at the present. What is apparent is that the SSC exposure in the present study is effective at yielding performance gains at an age corresponding with maturation and ineffective at an age when such maturation slows. The implication is that parameters of chronic SSC exposure (e.g. frequency of sessions and number of repetitions) should be investigated to promote adaptation in strength gains at all stages of life.

The parameters of SSC exposure of the present study were designed with the aim of inducing muscle mass and strength gains, especially at a young age [8]. Despite this intent, improvement in the recovery from fatigue was the only positive outcome observed for 6 month old rats. Improved fatigue recovery following chronic contraction exposure in young adult rats has been reported previously as well $[28,29]$. In the present study, we established that this SSC exposure-induced improvement was accompanied by a shift in fiber type distribution (i.e. type IIb to type IIx fibers) which was most pronounced for the 6 month age group relative to the other age groups. An increase in fatigue recovery accompanied by decreased type IIb fiber composition has been observed in a study regarding endurance training of human subjects [30]. In that study and the present investigation, the extent of fatigue during contractions was not improved. This is consistent with the notion that recovery from fatigue may be more sensitive than fatigue during contractions to fiber type shifts induced by specific regimes (e.g. types of SSC exposure or endurance training) [30].

Inclusion of the intermediate age groups provided the opportunity to determine what alterations in the adaptive response were due to maturation vs. aging per se. Since 6 month old rats were at an age when maturation is nearing completion as compared with that of the 3 month old rats, the adaptation in fatigue recovery without adaptation in strength was likely attributable to maturation status rather than the aging process. Altered hormonal, muscle architectural, and neural changes with maturation potentially underlie altered contractioninduced responses upon reaching young adulthood [27]. Consistent with reports demonstrating that the effects of aging can be detected long before the onset of chronic 
disease, the present study identified further decrements in SSC-induced adaptation at late adulthood (27 months of age) before the onset of age-related sarcopenia and weakness $[11,12]$. A substantial amount of research has been focused on comparing two age groups, typically an old age group vs a much younger age group, in regards to age-related deficiencies in performance $[4,5,8,10,16$, 17]. Based on the current findings, additional focus should be dedicated to studies which compare additional intermediate age groups to identify key regulatory factors and appropriate SSC regimes to account for maturationand aging-related alterations in muscle adaptive capacity.

\section{Acknowledgments}

This study was supported by internal funds at the National Institute for Occupational Safety and Health.

\section{Publication Disclaimer}

The findings and conclusions in this report are those of the author(s) and do not necessarily represent the views of the National Institute for Occupational Safety and Health.

\section{References}

[1] Lauretani F, Russo CR, Bandinelli S, Bartali B, Cavazzini C, Di Iorio A, et al. (2003). Age-associated changes in skeletal muscles and their effect on mobility: an operational diagnosis of sarcopenia. Journal of applied physiology, 95: 1851-1860

[2] Lovering RM, Brooks SV (2014). Eccentric exercise in aging and diseased skeletal muscle: good or bad? Journal of applied physiology, 116: 1439-1445

[3] Baker BA, Cutlip RG (2010). Skeletal muscle injury versus adaptation with aging: novel insights on perplexing paradigms. Exercise and sport sciences reviews, 38: 10-16

[4] Raue U, Slivka D, Minchev K, Trappe S (2009). Improvements in whole muscle and myocellular function are limited with high-intensity resistance training in octogenarian women. Journal of applied physiology, 106: 1611-1617

[5] Greig CA, Gray C, Rankin D, Young A, Mann V, Noble B, et al. (2011). Blunting of adaptive responses to resistance exercise training in women over $75 y$. Experimental gerontology, 46: 884-890

[6] Vaczi M, Nagy SA, Koszegi T, Ambrus M, Bogner P, Perlaki G, et al. (2014). Mechanical, hormonal, and hypertrophic adaptations to 10 weeks of eccentric and stretch-shortening cycle exercise training in old males. Experimental gerontology, 58: 69-77

[7] Komi PV (2000). Stretch-shortening cycle: a powerful model to study normal and fatigued muscle. Journal of biomechanics, 33: 1197-1206

[8] Cutlip RG, Baker BA, Geronilla KB, Mercer RR, Kashon ML, Miller GR, et al. (2006). Chronic exposure to stretch-shortening contractions results in skeletal muscle adaptation in young rats and maladaptation in old rats. Applied physiology, nutrition, and metabolism $=$ Physiologie appliquee, nutrition et metabolisme, 31: 573-587

[9] Baker BA, Hollander MS, Kashon ML, Cutlip RG (2010). Effects of glutathione depletion and age on skeletal muscle performance and morphology following chronic stretch-shortening contraction exposure. European journal of applied physiology, 108: 619-630

[10] Ryan MJ, Dudash HJ, Docherty M, Geronilla KB, Baker BA, Haff GG, et al. (2008). Aging-dependent regulation of antioxidant enzymes and redox status in chronically loaded rat dorsiflexor muscles. The journals of gerontology. Series A, Biological sciences and medical sciences, 63: 1015-1026

[11] Belsky DW, Caspi A, Houts R, Cohen HJ, Corcoran DL, Danese A, et al. (2015). Quantification of biological aging in young adults. Proceedings of the National Academy of Sciences of the United States of America,

[12] Cooper R, Bann D, Wloch EG, Adams JE, Kuh D (2015). "Skeletal muscle function deficit" in a nationally representative British birth cohort in early old age. The journals of gerontology. Series A, Biological sciences and medical sciences, 70: 604-607

[13] Geronilla KB, Miller GR, Mowrey KF, Wu JZ, Kashon ML, Brumbaugh K, et al. (2003). Dynamic force responses of skeletal muscle during stretch-shortening cycles. Eur. J. Appl. Physiol., 90: 144-153

[14] Baker BA, Hollander MS, Mercer RR, Kashon ML, Cutlip RG (2008). Adaptive stretch-shortening contractions: diminished regenerative capacity with aging. Applied physiology, nutrition, and metabolism = Physiologie appliquee, nutrition et metabolisme, 33: 1181-1191

[15] Bloemberg D, Quadrilatero J (2012). Rapid determination of myosin heavy chain expression in rat, mouse, and human skeletal muscle using multicolor immunofluorescence analysis. PloS one, 7: e35273

[16] Stewart VH, Saunders DH, Greig CA (2014). Responsiveness of muscle size and strength to physical training in very elderly people: a systematic review. Scandinavian journal of medicine \& science in sports, 24: e1-10

[17] Kosek DJ, Kim JS, Petrella JK, Cross JM, Bamman MM (2006). Efficacy of 3 days/wk resistance training on myofiber hypertrophy and myogenic mechanisms in young vs. older adults. Journal of applied physiology, 101: 531-544

[18] Ryan MJ, Dudash HJ, Docherty M, Geronilla KB, Baker BA, Haff GG, et al. (2010). Vitamin E and C supplementation reduces oxidative stress, improves antioxidant enzymes and positive muscle work in chronically loaded muscles of aged rats. Experimental gerontology, 45: 882-895

[19] Murlasits Z, Cutlip RG, Geronilla KB, Rao KM, Wonderlin WF, Alway SE (2006). Resistance training increases heat shock protein levels in skeletal muscle of 
young and old rats. Experimental gerontology, 41: 398406

[20] Lynch GS, Hinkle RT, Chamberlain JS, Brooks SV, Faulkner JA (2001). Force and power output of fast and slow skeletal muscles from mdx mice 6-28 months old. The Journal of physiology, 535: 591-600

[21] Thom JM, Morse CI, Birch KM, Narici MV (2005). Triceps surae muscle power, volume, and quality in older versus younger healthy men. The journals of gerontology. Series A, Biological sciences and medical sciences, 60: 1111-1117

[22] Graber TG, Kim JH, Grange RW, McLoon LK, Thompson LV (2015). C57BL/6 life span study: agerelated declines in muscle power production and contractile velocity. Age, 37: 9773

[23] Yarasheski KE, Lemon PW, Gilloteaux J (1990). Effect of heavy-resistance exercise training on muscle fiber composition in young rats. Journal of applied physiology, 69: 434-437

[24] Hellyer NJ, Nokleby JJ, Thicke BM, Zhan WZ, Sieck GC, Mantilla CB (2012). Reduced ribosomal protein s6 phosphorylation after progressive resistance exercise in growing adolescent rats. Journal of strength and conditioning research / National Strength \& Conditioning Association, 26: 1657-1666

[25] Volek JS, Gomez AL, Scheett TP, Sharman MJ, French DN, Rubin MR, et al. (2003). Increasing fluid milk favorably affects bone mineral density responses to resistance training in adolescent boys. Journal of the American Dietetic Association, 103: 1353-1356

Gilbert KL, Stokes KA, Hall GM, Thompson D (2008). Growth hormone responses to 3 different exercise bouts in 18- to 25- and 40- to 50-year-old men. Applied physiology, nutrition, and metabolism $=$ Physiologie appliquee, nutrition et metabolisme, 33: 706-712

[27] Pesta D, Thaler A, Hoppel F, Macek C, Schocke M, Burtscher M (2014). Effects of a 10-week conventional strength training program on lower leg muscle performance in adolescent boys compared to adults. The Journal of sports medicine and physical fitness, 54: 147-153

[28] Willems ME, Stauber WT (2000). Effect of resistance training on muscle fatigue and recovery in intact rats. Medicine and science in sports and exercise, 32: 18871893

[29] Willems ME, Stauber WT (2002). Fatigue and recovery at long and short muscle lengths after eccentric training. Medicine and science in sports and exercise, 34: 17381743

[30] Sinacore DR, Coyle EF, Hagberg JM, Holloszy JO (1993). Histochemical and physiological correlates of training- and detraining-induced changes in the recovery from a fatigue test. Physical therapy, 73: 661667 